\title{
Lifetime-Aware Hierarchical Wireless Sensor Network Architecture with Mobile Overlays
}

\author{
Maryam Soltan, Morteza Maleki, and Massoud Pedram \\ University of Southern California \\ \{soltan,morteza,pedram\}@usc.edu
}

\begin{abstract}
Recent technological advances have led to the emergence of small battery-powered sensors with considerable, albeit limited, processing and communication capabilities. Wireless sensor networks have therefore gained considerable attention in applications where spatially distributed events are to be monitored with minimal delay. We present and analyze a hierarchical wireless sensor network with mobile overlays, along with a mobility-aware multi-hop routing scheme, in order to optimize the network lifetime, delay, and local storage size. Fixed event aggregation relays and mobile relays are used to collect events from the sensors and send them to a central base station. We analyze the effects of various system parameters on the network performance, and formulate a convex optimization problem for maximizing the network lifetime subject to constraints on local storage, delay, and maintenance cost. Network behavior is studied and analytical results are validated through extensive simulations.
\end{abstract}

\section{INTRODUCTION AND PRIOR WORK}

A key technical challenge in most Wireless Sensor Networks $(W S N)$ is how to effectively deploy and coordinate the sensors and how to manage data collection in order to achieve a minimum end-to-end delay, a maximum Monitoring Lifetime $(M o L)$, and a desired Quality of Monitoring $(Q o M)$. MoL is duration of time that monitoring service is provided while QoM captures achievable performance and accuracy of data. Both of these parameters depend on sensor initial energy, deployment density, placement, sampling rate, network connectivity, and communication cost.

Several WSN architectures have been proposed for various applications. A simple one is a flat architecture where sensors also act as routers and transfer data through multi-hop routing. To better utilize limited communication and computation power of the sensors, a two-tier network may be employed [1], where more powerful fixed or mobile relays are used to collect and route the sensor data. With fixed relays, however, non-uniform sensor-torelay distances require that the sensors closer to relays also transmit information originated from other sensors. This leads to a heterogeneous network with a non-uniform depletion rate of energy sources and hence shorter lifetime. Since regular battery replacement in a large network is not a feasible solution, we consider mobile relays. Mobility assisted data collection in different applications of WSN has recently been discussed in the literature [2-7]. Most of the prior work, however, has been focused on single-hop communication to the mobile node.

Our main contributions in this paper are to analyze the effect of mobility in a multi-tiered wireless sensor network under multihop routing and to present a mobility-aware routing scheme. Specifically, we consider a WSN architecture, which consists of three hierarchical levels of sensors, Event Aggregation Relays $(E A R)$, and Mobile Aerial Infrastructure overLay (MAIL) nodes. The sensors are deployed in spatially distributed groups where each group is assigned a fixed $E A R$ node. $E A R$ nodes are low power event processing and data relay devices, which collect data through single-hop communication with the sensors, process local events and transmit data packets, through multi-hop wireless communication, to the MAIL nodes. Finally, mobile MAIL nodes forward data to the base station. Using aerial vehicles is especially helpful when terrain contains many blockages, e.g., mountainous regions, and ground-to-ground communication over large distances is not efficient due to large signal attenuation.

We exploit the mobility to increase $M o L$ by reducing and balancing power consumption of sensor and EAR nodes. More precisely, we analyze the effects of velocity and communication power of MAILs on network lifetime, delay, and local storage buffers. Furthermore we propose a multi-hop routing scheme in the $E A R$ layer, which considers mobility and queuing delays, in order to improve network performance, size of buffers, and network maintenance.

First we discuss our network structure and parameters. Then in sections III and IV we present our routing scheme as well as our analytical results on the effects of various system parameters on network performance. In section $\mathrm{V}$ we show how the constrained lifetime maximization can be formulated as a convex optimization problem. Finally, we present our system behavioral studies as well as our simulation platform and simulation results.

\section{NETWORK STRUCTURE AND PARAMETERS}

Figure 1 depicts our hierarchical WSN architecture comprising of sensor, EAR, and MAIL nodes. Sensors are deployed on the boundaries of a closed region, such that a minimum $Q O M$ is achieved. Possible applications of this type of sensor deployment are oil and gas refinery protection, borders or battlefield surveillance, oil pipeline safeguarding, etc. Sensors are grouped based on their location and their data reporting rate such that the groups have a balanced data generation rate. Each sensor in a group directly communicates to its associated EAR. EAR nodes are fixed low power data relay nodes that collect data and route it to a nearby MAIL node, and hence reduce communication burden on sensors and add more power control flexibility. Finally each $M A I L$ directly forwards the data to the central base station.

Sensors are very low power devices with short-range transmitters, while EAR nodes are battery-powered devices with capability of routing and communicating with aerial vehicles. MAIL trajectory is determined based on the location and the data traffic of EAR nodes. In this paper we assume that deployment

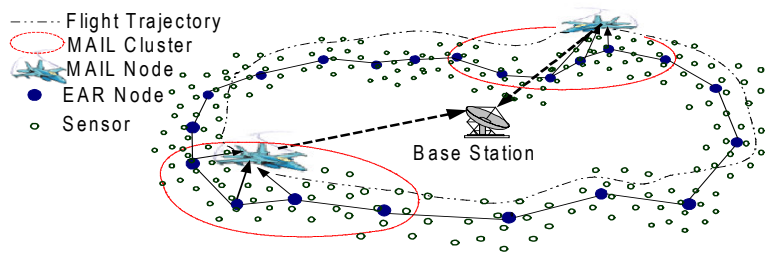

Figure 1: Hierarchical Sensor Network with Mobile Overlays. 
and trajectories of each level of the network are given and we study how mobility in the MAIL level and multi-hop routing in the $E A R$ level can affect the overall network performance.

\section{Network Parameters and Definitions}

Some of network parameters, notation, and their descriptions are provided in Table 1. Network lifetime is the duration of time before any of the sensor groups dies. This occurs when the sensors within the group fail to provide a minimum $Q o M$ or when the associated EAR node runs out of battery. In this paper, we focus on the EAR level of the network (issues related to sensor level failures fall outside the scope of the present work.) The overall MoL depends on network lifetime and lifetime of aerial vehicles.

Let recurrence cycle denote the time period for an $E A R$ to be visited by two consecutive MAILs and revisiting cycle represent the time period for a particular $E A R$ to be re-visited by the same $M A I L$. To obtain network lifetime, given the battery capacity of each $E A R$ node, energy consumption during one recurrent cycle should be calculated. Energy consumption for wireless transmission is $\varepsilon=e_{t} d^{\beta}$ [8], where $d$ is distance and $e_{t}$ refers to energy dissipation for transmitting unit of data over unit of distance. Two-ray propagation model may be assumed for $E A R$ to-EAR communication, while EAR-to-MAIL communication may be assumed to follow the free-space model. Moreover, MAIL is typically equipped with higher gain antennas and thus $e_{t}$ would be smaller for EAR-to-MAIL communication compared to communication between two EAR nodes.

\section{ROUTING SCHEME}

Without any delay and buffer size constraints, the optimal routing strategy for minimizing energy would be single-hop routing whereby an EAR node locally stores its data packets when it is outside the radio coverage of any MAIL and transmits its stored data when it is in the coverage region. However, due to latency and buffer size constraints and MAIL velocity limitations, and to avoid excessive packet losses, we propose a mobility-aware multi-hop routing scheme at the EAR level.

Each MAIL acts as a cluster-head for a set of EAR nodes. Due to mobility, EAR nodes within each cluster change over time. However, at any instance of time, each $E A R$ belongs to only one cluster and forwards its data towards one MAIL. To select the routing path for a specific $E A R$, first a $M A I L$ should be selected. Selecting closest MAIL clearly leads to less energy dissipation and less delay. EAR nodes form two chains around each MAIL. In one chain data packets are sent in the same direction as departing $M A I L$ while in the other, data is forwarded toward the approaching MAIL. We call these chains backward and forward chains, respectively (cf. Figure 2). We find the closest MAIL based on the dynamic distance, which is measured in terms of the Dynamic Hop Count $(D H C)$, that is, the actual number of hops

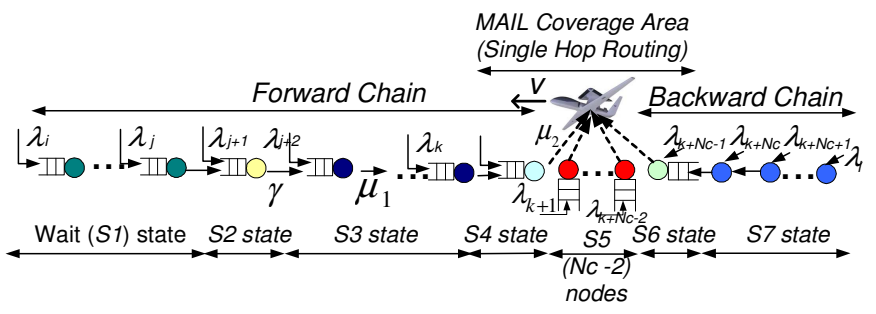

Figure 2: Cluster of EAR nodes, a MAIL node as a cluster head, link arrival and service rates, and state transition during recurrent cycle.
Table 1: Notations and their descriptions

\begin{tabular}{|c|c|c|c|}
\hline & Description & & Description \\
\hline$N$ & Number of $E A R$ in network & $M$ & Number of MAlLs \\
\hline$\lambda_{\mathrm{j}}$ & EAR j Aggregation Rate & $E_{0}$ & Initial Energy of an EAR node \\
\hline $1 / \mu_{1}$ & Avg. Link Delay for EAR-level & $1 / \mu_{2}$ & Avg. Link Delay for EAR-MAIL \\
\hline$\beta$ & Path Loss Exponent & $e_{t}$ & Unit of Transmission Energy \\
\hline$E_{M}$ & MAIL Initial Energy & $H$ & Hop Count Bound \\
\hline$v$ & MAIL Velocity & $T_{M}$ & MAIL Recurrent Cycle \\
\hline$R_{C}$ & MAIL Coverage Distance & \multirow{2}{*}{$N_{C}$} & \multirow{2}{*}{$\begin{array}{l}\text { Avg. number of EAR nodes } \\
\text { under MAIL Coverage }\end{array}$} \\
\hline $\bar{W}$ & Avg. Waiting Time in Queue & & \\
\hline$C_{M}$ & MAIL Re-Charging Cost & $C_{\max }$ & Maximum cost for UAV service \\
\hline$D_{\text {net }}$ & Avg. Network Delay & $D_{\max }$ & Delay Constraint \\
\hline$T_{n e t}$ & Network Lifetime & $T_{s y s}$ & System Lifetime \\
\hline
\end{tabular}

needed to transfer data from an EAR to a MAIL. Note that due to mobility and routing delays associated with transmission, propagation and queuing, $D H C$ differs from the Initial Hop Count (IHC) which is the number of hop counts that the EAR node will initially see at the starting time of transmission.

To minimize total energy consumption subject to latency and buffer size constraint, we propose a Bounded Hop-count Routing $(B H R)$ strategy such that an EAR starts forwarding its data to a $M A I L$ when it needs less than $H$ actual hops for data transmission. Otherwise, it moves to a wait state and stores data until a MAIL becomes reachable again by $H$ hops or less.

\section{IV.SYSTEM ANALYSIS}

We analyze $B H R$ routing strategy to determine network delay, buffer size, and lifetime. Each $E A R$ periodically experiences seven states based on $D H C$ to closest MAIL (cf. Figure 2). Let $t_{\text {link } 1}=\bar{W}+\left(1 / \mu_{1}\right)$ be total delay due to communication and average waiting time in queue, and $\bar{l}$ be the average distance that a MAIL travels between two EARs. Also note that Recurrence cycle time is the shortest period of time that a particular EAR node returns to any one of these states. By definition, a packet needs to be routed for $(D H C-1)$ hops before reaching the single hop coverage area on the respective chain. It can thus be shown that $D H C$ and $I H C$ for forward and backward chains are related as:

$$
(D H C-1)=(I H C-1) /(1 \pm \Gamma), \quad \Gamma \triangleq t_{\text {link } 1} / \Delta t, \Delta t \triangleq \bar{l} / v
$$

\section{A. Queuing Analysis}

State definitions and transition times are presented in Table 2. Let $\Delta t$ denote the average time needed for $M A I L$ to fly the physical distance of one hop. Furthermore, after time $\Delta t_{F}$ and $\Delta t_{B}, D H C$ for an $E A R$ changes one hop for forward and backward chains, respectively. Notice that the state transition time calculation considers the effect of queuing delays. Knowledge of the arrival, departure, and communication service rates of data packets in each state enables us to calculate queue size and subsequently calculate the average waiting time in each queue.

Let $\lambda$ be the aggregate arrival rate of the packets from each group of sensors to their associated $E A R$. Furthermore, let $\mu_{I}$ and $\mu_{2}$ respectively be the service rates for $E A R$-level and $E A R$ to $M A I L$ links. Due to higher contention around a $M A I L$, longer time is typically required to access the channel for successful transmission of a packet to $M A I L$ and hence $\mu_{2}<\mu_{1}$.

For an $E A R$ node $i, Q_{i}(t), A_{i}(t)$, and $U \mathrm{i}(\mathrm{t})$ respectively denote queue size, arrival rate and departure rate to/from node $i$ at time $t$. 
Table 2: State definitions and state durations

\begin{tabular}{|c|c|c|c|}
\hline State & $\begin{array}{c}\text { Minimum } \\
\text { Dynamic Hop } \\
\text { Count } \\
(M D H C)\end{array}$ & $\begin{array}{c}\text { Status of } \\
\text { receiving data } \\
\text { from other } \\
\text { sensors }\end{array}$ & $\begin{array}{c}\text { States' Transition times } \\
\Delta t \triangleq \bar{l} / v \quad, \quad \Gamma \triangleq t_{\text {link } 1} / \Delta t, \\
\Delta_{F} \triangleq(1-\Gamma) \Delta t, \Delta_{B} \triangleq(1+\Gamma) \Delta t\end{array}$ \\
\hline$S_{1}$ & $M D H C>H$ & None,(on wait) & $T_{1}=T_{M}-\sum_{i=2}^{7} T_{i}$ \\
\hline$S_{2}$ & $M D H C=H$ & None & $T_{2}=\Delta t_{F}$ \\
\hline$S_{3}$ & $1<M D H C<H$ & Receiving & $T_{3}=(H-2) \cdot \Delta t_{F}$ \\
\hline$S_{4}$ & $M D H C=1$ & Receiving & $T_{4}=\Delta t_{F}$ \\
\hline$S_{5}$ & $M D H C=1$ & None & $T_{5}=\left(N_{c}-2\right) \cdot \Delta t$ \\
\hline$S_{6}$ & $M D H C=1$ & Receiving & $T_{6}=\Delta t_{B}$ \\
\hline$S_{7}$ & $1<M D H<H$ & Receiving & $T_{7}=(H-1) \cdot \Delta t_{B}$ \\
\hline
\end{tabular}

Time reference, $t=0$, is when node $i$ enters state $S_{l}$. Graphs capturing sample time variations of $A_{i}(t), U_{i}(t)$ and $Q_{i}(t)$ are provided in Figure 3 . Note that these waveforms can change based on the relative values of of $H, \lambda, \mu_{1}$, and $\mu_{2}$. For instance, as shown in Figure 3(c), there is no queue built-up in states $S_{6}$ and $S_{7}$. This happens when $H . \lambda<\mu_{2}<\mu_{l}$.

The relation between $A_{i}(t), U \mathrm{i}(\mathrm{t})$ and $Q_{i}(t)$ is as follows:

$U_{i}(t)=\left\{\begin{array}{l}\mu_{1} \text { or } \mu_{2} \text { if } Q_{i}(t)>0 \\ A_{i}(t) \quad \text { if } Q_{i}(t)=0\end{array} \quad\left(\right.\right.$ For $\left.t>T_{1}\right)$ otherwise $U_{i}(t)=0$

The transition points of the queue length curve, $Q_{i}($.$) , are$ denoted by $L_{i}$ and $T_{j}$ denotes duration of time that node $i$ spends in state $S_{i}$. From Little's theorem, the average waiting time at node $i$ is $\bar{W}_{i}=\bar{Q}_{i} / \bar{A}_{i}$ and the average waiting time over all nodes would be $\bar{W}$. Given $\Gamma \triangleq t_{\text {link } 1} / \Delta t$ and $t_{l i n k 1}=\bar{W}+\left(1 / \mu_{1}\right)$, we have a system of two equations for $\Gamma$ and $\bar{W}$ to obtain the average waiting time for a packet in each buffer.

Delay consists of the queuing and link delays. Therefore, for a network with average hop count, $\bar{h}$, the average end-to-end delay, $D_{\text {net }}$, can be calculated as:

$D_{n e t}=\bar{h} \cdot \bar{W}+\frac{(\bar{h}-1)}{\mu_{1}}+\frac{1}{\mu_{2}}$

Using the above equation, $D_{\text {net }}$ can be shown to be proportional to $1 /\left(v H^{2}\right)$ and inversely proportional to the number of MAILs i.e. $D_{n e t} \propto(1 / M)$.

Given the average $D H C$, average energy dissipation for routing packets can be obtained. Because energy model parameters of EAR-to-EAR and EAR-to-MAIL communications are assumed to be different, we separately calculate the average energy needed for data transfer to an $E A R$ within coverage area, and the energy for sending a packet from that node to the MAIL. Let $\bar{E}$ denotes the average energy dissipation for routing a packet and $e_{E}$ and $e_{M}$ respectively denote average energy consumptions for EAR-to-EAR and EAR-to-MAIL transmission. Hence,

$\bar{E}=(\bar{h}-1) e_{\mathrm{E}}+e_{M}$

where $e_{E}$ and $e_{M}$ respectively denote average energy consumptions for EAR-to-EAR and EAR-to-MAIL transmission:

$e_{M}=e_{t 2} \cdot R_{C}{ }^{\beta 2}, \quad e_{E}=e_{t 1} \cdot \bar{d}^{\beta 1}$

where $\bar{d}$ is the average distance between two EARs. Average number of packets generated during the lifetime of the network is equal to $N \lambda T_{\text {net }}$. Hence,

$\bar{E} .\left(N \lambda T_{n e t}\right)=N E_{0} \Rightarrow T_{n e t}=E_{0} /(\lambda \bar{E})$

It can be shown that the network lifetime, $T_{n e t}$, (i.e., excluding $M A I L$ endurance) is in fact independent of velocity and is inversely proportional to the square of maximum hop-count, i.e. $T_{n e t} \propto\left(1 / H^{2}\right)$.

Another constraint is the size of the local buffer of each $E A R$ node. When the internal queue size of a node, $B_{p}$, exceeds $B_{\max }$, overflow occurs. Therefore, in order to avoid packet loss, the peak value of the queue size should be upper-bounded by $B_{\max }$.

\section{NETWORK OPTIMIZATION PROBLEM}

We derived delay and network lifetime as functions of the network parameters, i.e., velocity and hop-count bound and MAIL coverage distance. For the BHR routing scheme these parameters should be optimally selected in order to maximize lifetime subject to delay, buffer size and MAIL recharging cost constraints.

The overall life time of system is $T_{s y s}=\operatorname{Min}\left(T_{n e t}, \quad c . T_{e}\right)$, where $T_{e}=\alpha E_{M} /\left(\rho v^{3}\right)$ is the aerial vehicle endurance time, $\rho$ is air density and factor $\alpha$ depends on the fuel used for providing energy [9]. Also $c$ is number of times a MAIL leaves for recharging. Hence, the optimization problem can be presented as:

$$
\begin{aligned}
& \underset{v, H, R_{C}}{\operatorname{Max}} \operatorname{Min}\left(T_{n e t}, c \cdot T_{e}\right) \\
& \text { s.t. } \quad D_{n e t} \leq D_{\max }, \quad B_{p} \leq B_{\max }, \quad c \cdot C_{M} \leq C_{\max }
\end{aligned}
$$

To solve this Max-Min optimization problem, lets define $f$ $=1 / T_{\text {sys. }}$. Hence, based on definition of $T_{\text {sys }}, f \geq 1 / T_{\text {net }}$ and $f \geq 1 / c T_{e}$. Therefore the objective can be transformed into the following convex standard epigraph form:

$$
\begin{aligned}
& \underset{v, H}{M} \operatorname{in}_{R_{C}} f \\
& \text { s.t. } \quad \lambda \bar{E}-f \cdot E \leq 0 \quad, \quad \rho \cdot v^{3}-f \cdot c \cdot \alpha \cdot E_{M} \leq 0
\end{aligned}
$$

The objective in (8) is linear. Furthermore, the constraints have linear or polynomial forms with positive coefficients with respect to the variables. One can thus prove the convexity of the optimization problem by showing that the second derivatives of constraints with respects to the variables are positive.

\section{Simulation PlatForm AND RESUlts}

We developed a sophisticated network simulator for the analysis of our network characteristics under the proposed mobility-aware bounded multi-hop routing scheme. While the system can be modeled as a network of $\mathrm{G} / \mathrm{G} / 1 / \mathrm{K}$ queues, we focused on $\mathrm{D} / \mathrm{D} / 1 / \mathrm{K}$ and $\mathrm{M} / \mathrm{M} / 1 / \mathrm{K}$ queues with vacation and variable service rates over the time. The simulations were performed for a sensor

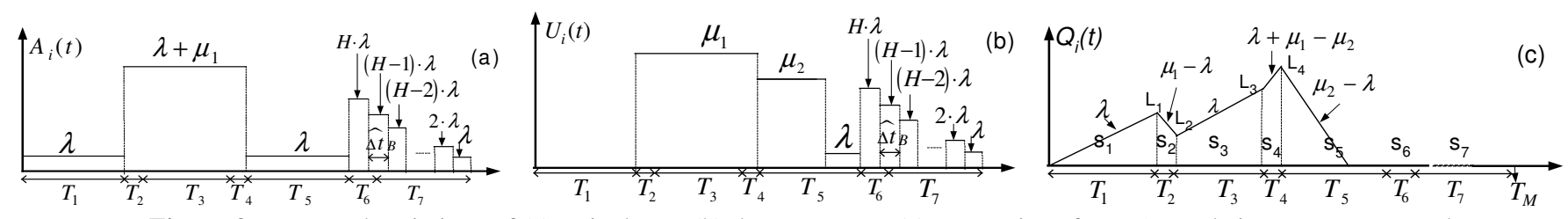

Figure 3: Temporal variations of (a) arrival rate, (b) departure rate, (c) queue size of an EAR node in a recurrence cycle. 

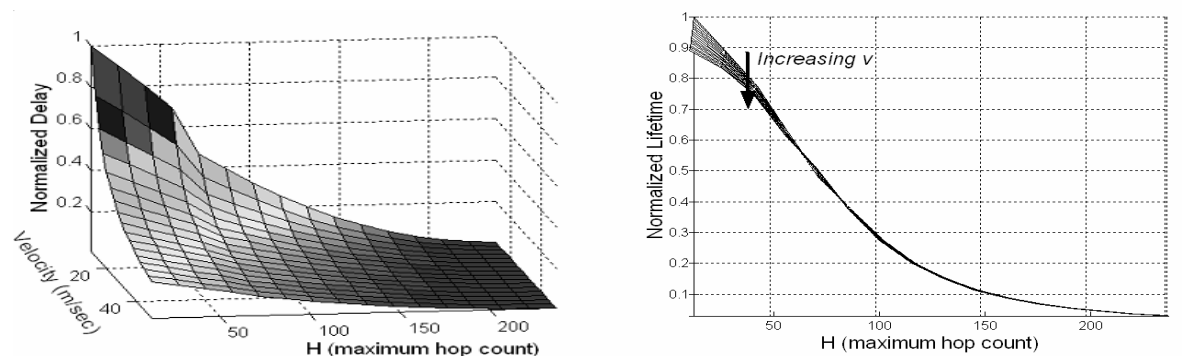

Figure 5: Effect of $v$ and $H$ on network delay and lifetime.

network with 100 EAR nodes randomly distributed on the borders of a closed area and two MAILs covering the overlay network. Path loss exponents follow free space and two ray models. Values of $e_{t}$ for EAR-to-EAR and EAR-to-MAIL transmissions are 0.0013 and 10 (pJoul $/ \mathrm{bit} / \mathrm{m} 2)$, respectively [8]. $\lambda$ is set to 0.3 packets per second. We evaluated the effects of hop count bound, MAIL communication coverage area and its velocity, and validated our analytical results through simulations.

Figure 4 shows sample queue size variations. Also as shown in Figures 5, and 6, as the maximum bound on the number of hops, $H$, increases, both the network delay and the lifetime are reduced. This clearly indicates the trade-off between delay and lifetime. Note that increasing MAIL velocity could reduce the overall system lifetime due to shorter MAIL endurance time. MAIL single-hop coverage area has similar effects as the maximum hop count on delay and network lifetime. Larger coverage area means more data transmission to the MAIL, which in turn implies lower delay. The sudden changes in some points with respect to $H$ are due to the characteristics of queuing backlogs.

\section{CONCLUSIONS}

In this paper, a novel lifetime and delay aware deployment strategy for a hierarchical WSN is investigated. A mobility-aware multi-hop routing scheme is proposed and detailed analysis is conducted to characterize network lifetime, end-to-end delay, and buffer size. A packet level simulator is built to validate the analytical results. Effect of velocity and hop count bound on lifetime and delay are studied. This study shows that network delay is inversely proportional to the velocity of MAILs, whereas the effect of velocity on network lifetime is small. However, considering the mobile overlay, increasing velocity affects $M o L$. Moreover, our bounded dynamic hop count routing introduced a trade of between delay and lifetime witch can be controlled using the hop count bound. Note that in our scheme, we do not route data unless $D H C$ is less than the hop count bound and thus selecting a feasible buffer size will avoid packet loss.

\section{REFERENCES}

[1] A. Iranli, M. Maleki, M. Pedram, "Energy efficient strategies for deployment of a two-level wireless sensor network," Proc. of the International Symposium on Low Power Design (ISLPED), 2005.

[2] Q. Li and D. Rus, "Sending messages to mobile users in
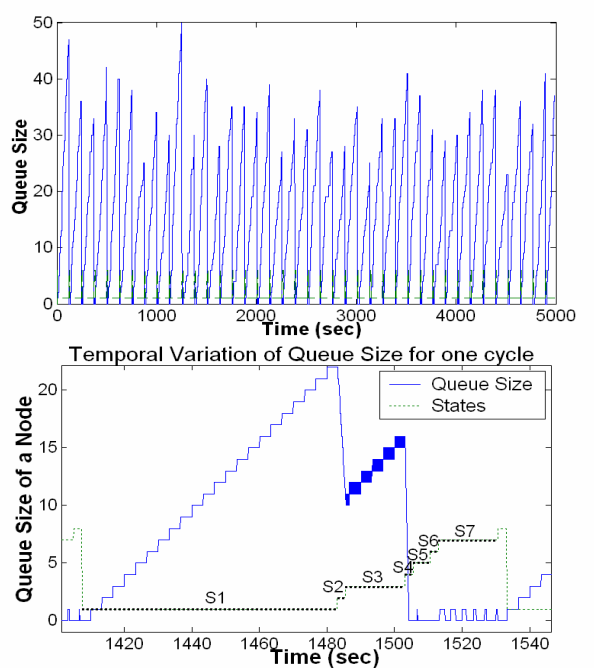

Figure 4: Simulated temporal queue size variation.

disconnected ad-hoc wireless networks," Proc. of MobiCom, 2000.

[3] R. Shah, S. Roy, S. Jain, and W. Brunette, "Data MULEs: Modeling a Three-tier Architecture for Sparse Sensor Networks," IRS-TR-03-001, Jan. 21, 2003.

[4] S. Jain, R. C. Shah, G. Borriello, W. Brunette, and S. Roy, "Exploiting mobility for energy efficient data collection in sensor networks," Proc. of Modeling and Optimization in Mobile, Ad Hoc and Wireless Networks (WiOpt), 2004.

[5] W. Zhao, M. Ammar, and E. Zegura, "A message ferrying approach for data delivery in sparse mobile ad hoc networks," Proc. of MobiHoc, 2004.

[6] A. Kansal, A. A. Somasundara, D. D. Jea, M. Srivastava, and D. Estrin, "Intelligent fluid infrastructure for embedded networks," Proc. Of MobiSys, 2004.

[7] J. Luo and J.-P. Hubaux, "Joint mobility and routing for lifetime elongation in wireless sensor networks," Proc. of the 24th Conference of the IEEE Communications Societies, 2005.

[8] W.R.Heinzelman, A.Chandrakasan, and H. Balakrishnan, "An Application-Specific Protocol Architectures for Wireless Networks," IEEE Trans. On Wireless Communication, vol. 1, no. 4, 2002.

[9] Autonomous Vehicles in Support of Naval Operations, National Academies Press,

http://www.nap.edu/books/0309096766/html/200.html.
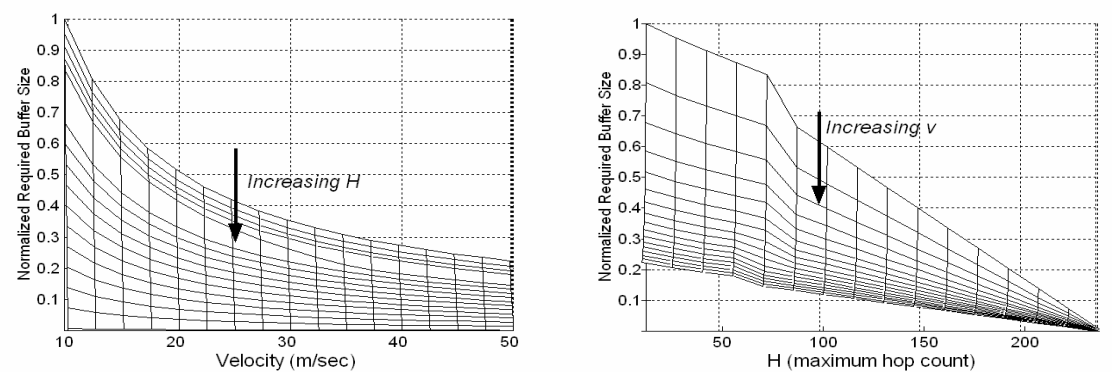

Figure 6: Effect of $v$ and $H$ on required buffer size. 